\title{
Operations of Online Advertising Services and Publisher's Option
}

\author{
Anand Srinivasan Changhyun Kwon* \\ Department of Industrial and Systems Engineering \\ University at Buffalo, the State University of New York
}

\begin{abstract}
We analyse the use of options for online advertisement publishers. By providing a discount or rewards to advertisers, publishers can utilize their uncertain service capacity, page-views, more efficiently. We use Generalized Nash Bargaining to study the feasibility of the option contract and solve for an optimal value for the option price. We compare the revenues and benefits from advertisements under the option contract, with those without the options using numerical studies. We also study the impact of pricing and other components in the game on the optimal option price, the publisher's revenues, and the advertiser's benefits from the advertisements.
\end{abstract}

Keywords: online advertising; Nash bargaining game; option contract

\section{Introduction}

Since its start in 1994, online advertising has gained steady popularity over the past 16 years. In the past decade, the online advertising industry grew from $\$ 4.6$ billion in 1999 to $\$ 22.7$ billion in 2009 (Internet Advertising Bureau, 2010). Online advertisements are broadly classified into four major categories: search based ads, display ads, classified ads, and email based ads. While search based ads have $47 \%$ market share in 2009, display ads have 22\% (Internet Advertising Bureau, 2010).

Being a relatively new concept, online advertising has gained attention from researchers. Evans (2008) discusses the evolution of online advertising industry in depth, including issues like trends in the industry and the privacy dilemma faced by the publishers. With the burst in online advertising, the intrusiveness of online advertising

\footnotetext{
${ }^{*}$ Corresponding Author: chkwon@buffalo.edu
} 
came into question. McCoy et al. (2007) measure this intrusiveness and come up with the result that ads which are different in content from the websites, have a greater impact on the viewer than ads similar in content. In addition, they conclude that pop-up ads and pop-under ads are more intrusive than in-line ads. Danaher and Mullarkey (2003) study factors affecting the performance of online display advertisements and show that the web page exposure duration (WPED) is a key determinant of brand recall .

There are also many research articles about the click-through rate (CTR), which is an important consideration in online advertising, which is the ratio of number of clicks on an advertisement to the number of views of the advertisement. Chatterjee et al. (2003) develop an analytical approach to model this measure of advertisement effectiveness. Drèze and Hussherr (2003) discuss the cause for plummeting CTRs and suggest that advertisers should rely more on traditional brand equity measures. Becker et al. (2007) develop different probabilistic models to model the CTR. Richardson et al. (2007) develop a model to predict the CTR for a new ad.

The initial concept of charging the advertiser for the ads was based on a pay per view (PPV) approach similar to the newspaper ads where the advertiser is charged per thousand views of his ad from a certain demography. However, as the market matured over time, this gave way to performance based pricing. The two major models based on this concept are pay per action (PPA) and pay per click (PPC). A PPA model essentially means that the advertiser is charged based on number of "actions" which can encapsulate anything beyond a click. While the number of actions is reported by the advertiser since actions occur in the advertiser's website, the number of clicks is reported by the publisher since clicks can be monitored by the publisher. Mahdian and Tomak (2008) discuss the advantages and issues associated with the PPA models.

In this paper, we study a less studied problem of how display advertisements should be priced. For display advertisements, PPV pricing methods are more popular, and PPA and PPC methods are less used. Because PPA and PPC methods are motivated by the idea that the number of display is not a good measure of the advertisement performance or does not indicate what the advertisers have to pay at least. Therefore, PPA and PPC methods are more appropriate to search advertisements. In display advertisement contracts, the publisher considers a display as a consumption of his page-view inventory, therefore the advertisement is charged based on the number of displays.

In 2009, performance based models accounted for $59 \%$ of total Internet ad revenues, while the PPV model accounted for $37 \%$ and the remaining $4 \%$ accounted for by hybrid models (Internet Advertising Bureau, 2010). Mangani (2004) considers an advertiser who chooses the PPV or PPC model for advertising and concludes that 
a mixture of PPV and PPC contracts may be the most optimal for the advertiser. Fjell (2009) revisits the same problem and draws a different conclusion: it is optimal for the publisher to opt for either PPV or PPC and not a combination of the two. More recently, Kwon (2009) considers a more detailed model for the optimal choice between PPV and PPC and reaches the same result as Fjell (2009) but with a different decision-making point. Roels and Fridgeirsdottir (2009) discuss the use of a dynamic optimisation model to maximise the publisher's revenues and they show that their model can significantly improve the revenue system of online advertisement publishers. Dinev et al. (2008) take into account the advertiser's trust in search engines, third party monitoring tools and filtering processes and develop a theoretical model to understand the advertiser's behaviour. Their results prove that attitudes and subjective norms influence the decision to advertise online using the PPC contracts. While many models have been developed for the revenue management of online advertising, the basic means of quantifying the costs remain restricted to PPV or PPC. Kumar and Sethi (2009) discuss the emergence of hybrid models in revenue management in online advertising industry and develop a model based on optimal control theory for optimal values of subscription fee and advertisement level.

In this paper, we present a model that presents some key components of display advertisement contracts. With advertisements on print, the number of views of the advertisements can be determined with a reasonable level of accuracy before the ad is actually printed. However, with online advertising, the number of page views is highly volatile. With PPV contracts, in case the number of page views of a particular website falls short of the contracted number of displays, the publisher needs to pay a penalty for every unfulfilled impression. This results in a loss in revenue proportional to the shortfall in number of page views. This is essentially the issue we seek to address in this model. Moon and Kwon (2011) introduce the concept of an option contract in online advertising and use the Generalized Nash Bargaining game approach to come up with the optimal option price. We implement a similar approach to solve the problem of loss in revenue for the publisher and loss in advertising effect for the advertiser due to low page views on a particular page. Game theoretic bargaining was introduced by Nash Jr. (1950) which provides the background for much of the work done on bargaining. Roth and Malouf (1979) analyse the role of information in bargaining by introducing the concept of Generalized Nash Bargaining. The applications of Generalized Nash Bargaining can be seen in several areas like supply chain management (Nagarajan and Sosic, 2008) and bandwidth allocation (Touati et al., 2006).

The notion of option contracts proposed in this paper has similar characteristics to other option or option-like concepts proposed in other industries: for example, the concepts of callable products (Gallego et al., 2008) and flexible products (Gallego and 
Phillips, 2004). A callable product is a service product which provides the seller an option to recall the product at a pre-specified recall price. The option we introduce in this article is similar in the sense that it allows the publisher to transfer some ads to the second website by paying the option price. A flexible product (a name used by Gallego and Phillips, 2004) is a service product with which customers do not know what exact service they will receive a priori. However, both callable and flexible products are more suitable to airlines and do not consider uncertainty in service capacity, which is unique to online advertising. Elmaghraby et al. (2009) discuss a case where retailer announces the price and the clearance price for the product at the beginning of the season and also provides an option to the consumer to reserve the product for purchase at clearance price at the end of the season if the product remains unsold.

The rest of the paper is organized as follows. In Section 2, we propose an option model and determine an optimal option price. We provide sensitivity studies in Section 3 to investigate what the impacts of some key parameters are for the option price, revenues of the publisher and benefits of the advertiser. In Section 4, we investigate what actually happens when the ad period is over and an uncertain variable is realized. Section 5 concludes this paper. Throughout the paper, we refer the publisher as him and the advertiser as her.

\section{The Option Model}

In this paper, we look at the PPV contracts in a situation where the publisher has two web pages Page 1 and Page 2. The advertiser has a preference of one web page over the other for advertisement. The contract between the publisher and the advertiser specifies a certain number of displays of the advertisement on Page 1 during the contract period. Let the initial contracted number of displays on Page 1 be $k$ for the advertising period. We explore the possibility of the advertiser offering an option, which gives the publisher the opportunity to shift the advertisement to Page 2 in case he is not able to accommodate the contracted number of displays on Page 1 . The number of page views of Page 1 is a random variable which we denote by $X_{1}$. Let $f_{1}(\cdot)$ and $F_{1}(\cdot)$ denote probability density function (pdf) and the cumulative distribution function (cdf) of $X_{1}$. The PPV on Page 1 is defined as $p_{1}$. We assume $p_{1}>p_{2}$.

When display advertisements are used, the advertiser aims to expose her advertisement for a certain times, usually to promote her brand value. Therefore the promised number $k$ is important. If the publisher do not promise a certain number of displays, the advertiser may not be able to achieve her objective in the advertisement campaign, because the publisher may opt out to display her advertisement for other advertisements with higher fee. 
In addition, in a display ad contract, the publisher promises $k$ for a specified time period. The reason is that the advertiser wants to advertiser her brand before a certain time, for example, introduction of new products before other competitors promote their new products, and announcement of a new event before the event date. When the publisher cannot display $k$ times within the contracted time, the publisher may display the unfulfilled displays in the next time period. Although we use another web-page as Page 2 in this paper, the same Page 1 in the next time period also can be regarded as Page 2. However, to display the advertisements in the same page in the next time period, the publisher must offer some discounts or other incentives to the advertiser. Therefore we assume $p_{1}>p_{2}$. However, in many cases, the publisher has other advertisement campaigns contracted in the next time period. Therefore if he carries over the unfulfilled number of displays to the next time period in the same page, it will impact other contracted advertisements and therefore make planning more complicated.

In the absence of the option, when $X_{1}$ turns out to be less than $k$, the publisher pays a penalty on the difference between $X_{1}$ and $k$. We assume this penalty cost per unfulfilled expression to be $h>0$. When $X_{1}$ exceeds $k$, the publisher can display advertisements from other networks advertisements. The network advertisements are a network of third-party display advertisements without contracted number of displays and time periods. The network advertisements are risk-free revenue generation opportunities for the publisher but their marginal revenue is much less than regular contracted advertisements. With the proposed option, the publisher gets the opportunity to shift some of the advertisements to Page 2 in case $X_{1}$ turns out to be less than $k$. By paying the option price, the publisher can try to save the penalty costs by pricing the transferred ads at a lower cost-per-impression (CPM) than the original one. The option can be exercised by the publisher at the end of the initial contract when the demand has been realized already. Thus by using the option, the publisher can display the remaining advertisements on Page 2 in the next period. We assume that there is space on Page 2 to do so.

Let us assume the option price to be $O_{P}$ and the CPM on Page 2 to be $p_{2}$. For the advertiser, the option provides a mode to achieve higher advertising effect through more number of page views than the case without option when number of page views on Page 1 falls short of the contracted value. To measure the utility of the advertisements to the advertiser, we define the utility of advertisements on Page 1 to be $U_{1}(\cdot)$ as a function of the number of displays of the advertisements on Page 1. Similarly, we define $U_{2}(\cdot)$. We assume that $U_{1}$ and $U_{2}$ are monotonically increasing functions, and Page 1 is preferred, i.e., $U_{1}(y) \geq U_{2}(y)$ for all $y \geq 0$.

The revenue with the option for the publisher would be the same as the cost with 
the option for the advertiser. We define the publisher's revenue with the option as $R_{O}$, which is expressed as:

$$
R_{O}=p_{1} \mathbb{E}\left[\min \left(X_{1}, k\right)\right]-O_{P}+p_{2} \mathbb{E}\left[\max \left(k-X_{1}, 0\right)\right]
$$

where $\mathbb{E}$ denotes expectations. We define the publisher's revenue without the option as $R_{W O}$, which is expressed as

$$
R_{W O}=p_{1} \mathbb{E}\left[\min \left(X_{1}, k\right)\right]-h \mathbb{E}\left[\max \left(k-X_{1}, 0\right)\right]
$$

where the first term represents the revenue from advertising in Page 1, and the second term represents the penalty fee received from the publisher if the publisher cannot display the promised number of displays $k$.

The net benefit of the advertising to the advertiser is defined as utility of the advertisements less the cost of advertising. We define the advertiser's net benefit from the option by $B_{O}$, which is expressed as:

$$
B_{O}=U_{1}\left(\mathbb{E}\left[\min \left(X_{1}, k\right)\right]\right)+U_{2}\left(\mathbb{E}\left[\max \left(k-X_{1}, 0\right)\right]\right)-R_{O}
$$

Similarly, we get define the benefit without the option as $B_{W O}$ and is expressed as

$$
B_{W O}=U_{1}\left(\mathbb{E}\left[\min \left(X_{1}, k\right)\right]\right)-R_{W O}
$$

For the sake of simplicity, we assume there exist extra page views on Page 2 to accommodate the additional advertisements if the option is exercised by the publisher.

We first determine the feasible values of the option price so that the option contract becomes viable for both the publisher and the advertiser.

Proposition 1. The feasible values of the option price, $O_{P}$, are given by:

$$
\left(p_{2}+h\right) \gamma-U_{2}(\gamma) \leq O_{P} \leq\left(p_{2}+h\right) \gamma
$$

where $\gamma=\mathbb{E}\left[\max \left(k-X_{1}, 0\right)\right]$.

Proof. If the option price is in the range $R_{O}-R_{W O} \geq 0$ and $B_{O}-B_{W O} \geq 0$, then both the publisher and the advertiser will consider the option contract as viable. From these two conditions, we obtain the conditions for when $O_{P}$ leads to an option contract agreement.

When the option price is negotiated, both the publisher and the advertiser can use the range specified in Proposition 1 for their guidelines. While any value of $O_{P}$ in the 
range in Proposition 1 will lead to an option contract agreement, both parties want to maximize the benefit from the option contract. We assume that the negotiation process between the publisher and the advertiser is a Generalized Nash Bargaining game. Both parties will consider the revenue/benefit from the option contract as well as the revenue/benefit when the option contract is not agreed. The optimal option price $O_{P}$ at equilibrium by Generalized Nash Bargaining is given by:

$$
O_{P}=\arg \max \left(R_{O}-R_{W O}\right)^{\alpha}\left(B_{O}-B_{W O}\right)^{1-\alpha}=\arg \max \theta
$$

where $\alpha$ and $1-\alpha$ are the relative negotiation powers of the publisher and the advertiser, $\alpha \in[0,1]$, and $\theta \equiv\left(R_{O}-R_{W O}\right)^{\alpha}\left(B_{O}-B_{W O}\right)^{1-\alpha}$. The differences $R_{O}-R_{W O}$ and $B_{O}-B_{W O}$ represents the relative benefits of the option contract, which both parties want to maximize in the negotiation. The solution of the Generalized Nash Bargaining game can be found as in the following proposition.

Proposition 2. The optimal option price determined by the Generalized Nash Bargaining game is

$$
O_{P}=\left(p_{2}+h\right) \gamma-\alpha U_{2}(\gamma)
$$

Proof. We will find an optimal value of $O_{P}$, which maximizes the value of $\theta$ using the first order and second order conditions. We consider $\max \log \theta$ and its first order condition

$$
\frac{\partial \log \theta}{\partial O_{P}}=0
$$

We obtain

$$
-\alpha\left(O_{P}+U_{2}(\gamma)\right)-\left(p_{2}+h\right) \gamma+(1-\alpha)\left[\left(p_{2}+h\right) \gamma-O_{P}\right]=0
$$

Finally, we have

$$
O_{P}=\left(p_{2}+h\right) \gamma-\alpha U_{2}(\gamma)
$$

We check the second order derivative:

$$
\frac{\partial^{2} \log \theta}{\partial O_{P}{ }^{2}}=-\frac{\alpha}{\left[\left(p_{2}+h\right) \gamma-O_{p}\right]^{2}}-\frac{1-\alpha}{\left[U_{2}(\gamma)+\left(p_{2}+h\right) \gamma-O_{p}\right]^{2}} \leq 0
$$

for all $\alpha \in[0,1]$, where $\gamma=\mathbb{E}\left[\max \left(k-X_{1}, 0\right)\right] \geq 0$. Thus, the $O_{P}^{*}$ obtained above, is a maxima and the proof is complete. Also, the optimal option price $O_{P}^{*}$ is feasible for all $\alpha \in[0,1]$.

The option price in Proposition 2 is a descriptive value. That is, while the negotiation process has many factors that affect the option price, we model the process using the notion of Generalized Nash Bargaining games and obtain the equilibrium solution 
(6). This paper proposes a notion of option contract in online display advertisements and examines its benefits using the descriptive equilibrium solution (6).

In this paper, we only consider when $O_{P} \geq 0$. That is, there is no incentive for the advertiser to consider the option contract if the advertiser has to pay extra fee to display the ad in Page 2. It would only happen when Page 2 has better utility for the advertiser. We consider

$$
O_{P}=\left(p_{2}+h\right) \gamma-\alpha U_{2}(\gamma) \geq 0 \quad \forall \gamma \geq 0
$$

We note that the value of $O_{P}$ is zero when $\gamma=0$. Therefore we must have

$$
\frac{\partial O_{P}}{\partial \gamma}=\left(p_{2}+h\right)-\alpha U_{2}^{\prime}(\gamma) \geq 0 \quad \forall \gamma \geq 0
$$

since we assumed $U_{2}$ is monotone. That is, to make the option contract viable, the marginal utility of the advertiser in Page 2 is small enough as

$$
U_{2}^{\prime}(\gamma) \leq \frac{p_{2}+h}{\alpha} \quad \forall \gamma \geq 0
$$

If the marginal utility in Page 2 is greater than what is specified in (12), the publisher would consider selling Page 2 as a separate advertising space. In addition, we can show that the optimal option price has directional changes with respect to negotiation powers.

Proposition 3. The option price $O_{P}$ is decreasing with the publisher's negotiation powers and increasing with advertiser's negotiation powers. That is,

$$
\frac{\partial O_{P}}{\partial \alpha} \leq 0 \quad \frac{\partial O_{P}}{\partial(1-\alpha)} \geq 0
$$

Proof. From the first order derivative of $O_{P}$ with respect to the publisher's negotiation power $\alpha$, we have,

$$
\frac{\partial O_{P}}{\partial \alpha}=-U_{2}(\gamma) \leq 0
$$

since $m_{2} \geq 0$ and $\mathbb{E}\left[\max \left(k-X_{1}, 0\right)\right] \geq 0$. Similarly, we obtain

$$
\frac{\partial O_{P}}{\partial(1-\alpha)}=U_{2}(\gamma) \geq 0
$$

Hence, the proof is complete.

Proposition 3 shows that the publisher can get the option contract at a lower option price, the higher his negotiation power is. 
The optimal option price, $O_{P}^{*}$, is independent of $p_{1}$, i.e. the initial CPM on Page 1. This indicates that the CPM on Page 1 is governed by the standard revenue management practices of the publisher. However, the CPM on Page $2, p_{2}$, and the penalty cost, $h$, does affect the optimal price. Increasing the CPM on Page 2 increases the optimal option price, $O_{P}^{*}$. In addition, the surplus revenue generated by the option to the publisher, $\left(R_{O}-R_{W O}\right)$, is $\alpha U_{2}(\gamma)$. This shows that the publisher's surplus is directly proportional to the advertiser's utility of ads on Page 2. Thus, unless the advertiser gains (in terms of utility of the ad) from the advertisements on Page 2, the publisher too does not have any incentive in using the option to transfer some ads to Page 2. The surplus generated by the option for the advertiser, $\left(B_{O}-B_{W O}\right)$, is $(1-\alpha) U_{2}(\gamma)$. We also notice that the ratio of the surplus generated by the option for the publisher and the advertiser simplifies to the ratio of their relative negotiation powers. That is:

$$
\frac{R_{O}-R_{W O}}{B_{O}-B_{W O}}=\frac{\alpha}{1-\alpha}
$$

\section{Sensitivity Study}

In this section, we study the sensitivity of the option price, revenues of the publisher and benefits of the advertiser to two parameters: the contracted number of displays $k$ and the standard deviation $\sigma$ of the number of page-view in Page 1. Since the option contract is proposed as a risk management tool for the publisher, we need to study the risk brought by changes in $k$ and $\sigma$. These two parameters are directly relevant to the risk of the publisher, because $k$ is compared with the uncertain variable $X_{1}$ and $\sigma$ presents how we are uncertain about $X_{1}$.

From the definitions and the optimal option value, we can obtain the following values:

$$
\begin{aligned}
O_{P} & =\left(p_{2}+h\right) \gamma-\alpha U_{2}(\gamma) \\
R_{O} & =p_{1} k-\left(p_{1}+h\right) \gamma+\alpha U_{2}(\gamma) \\
R_{W O} & =p_{1} k-\left(p_{1}+h\right) \gamma \\
B_{O} & =U_{1}(k-\gamma)+(1-\alpha) U_{2}(\gamma)-p_{1} k+\left(p_{1}+h\right) \gamma \\
B_{W O} & =U_{1}(k-\gamma)-p_{1} k+\left(p_{1}+h\right) \gamma
\end{aligned}
$$

where $\gamma=\mathbb{E}\left[\max \left(k-X_{1}, 0\right)\right]$. 


\subsection{Effects of the Contracted Number of Displays}

We investigate the effects of the contracted number of displays, $k$. We first note that

$$
\gamma=\mathbb{E}\left[\max \left(k-X_{1}, 0\right)\right]=k F_{1}(k)-\int_{0}^{k} x f_{1}(x) d x
$$

and

$$
\frac{\partial \gamma}{\partial k}=F_{1}(k)
$$

We obtain:

$$
\begin{aligned}
\frac{\partial O_{P}}{\partial k} & =\left(p_{2}+h\right) F(k)-\alpha U_{2}^{\prime}(\gamma) F(k) \\
\frac{\partial R_{O}}{\partial k} & =p_{1}-\left(p_{1}+h\right) F(k)+\alpha U_{2}^{\prime}(\gamma) F(k) \\
\frac{\partial R_{W O}}{\partial k} & =p_{1}-\left(p_{1}+h\right) F(k) \\
\frac{\partial B_{O}}{\partial k} & =U_{1}^{\prime}(k-\gamma)(1-F(k))+(1-\alpha) U_{2}^{\prime}(\gamma) F(k)-p_{1}+\left(p_{1}+h\right) F(k) \\
\frac{\partial B_{W O}}{\partial k} & =U_{1}^{\prime}(k-\gamma)(1-F(k))-p_{1}+\left(p_{1}+h\right) F(k)
\end{aligned}
$$

where $U_{1}^{\prime}$ and $U_{2}^{\prime}$ are first-order derivatives of $U_{1}$ and $U_{2}$ respectively. From (11), we observe that $\frac{\partial O_{P}}{\partial k} \geq 0$, that is, if the contract size is bigger, the option price is also bigger.

Let us consider (21). Without option contract, the publisher will try to maximize the revenue by choosing $k=F^{-1}\left(p_{1} /\left(p_{1}+h\right)\right)$ to make $\frac{\partial R_{W O}}{\partial k}=0$. However, the revenue with option contract is not maximised at such $k$ since $\frac{\partial R_{O}}{\partial k}$ at such $k$ is positive. Therefore with the option contract, the publisher can accept a higher number of displays. In this paper, we do not discuss how $k$ would be or should be determined. Rather, we argue that the contract size can be bigger with option contract and consequently it has potential to generate more revenue for the publisher. The behaviour of variables with varying $k$ is illustrated in Figure 1. While we use the parameters in Table 1, we vary $k$ and observe the behaviour of the option price, revenues of the publisher and benefits of the advertiser. As shown above, the option price increases as $k$ increases. In the computational results in this section, we assume linear utility functions $U_{1}(y)=m_{1} y$ and $U_{2}(y)=m_{2} y$. 
Table 1: Parameter values for simulation

\begin{tabular}{|c|c|c|c|}
\hline$p_{1}$ & 0.010 & $k$ & 100000 \\
\hline$p_{2}$ & 0.008 & $\alpha$ & 0.6 \\
\hline$m_{1}$ & 0.020 & $h$ & 0.020 \\
\hline$m_{2}$ & 0.010 & $X_{1}$ & $\sim N\left(135000,30000^{2}\right)$ \\
\hline
\end{tabular}

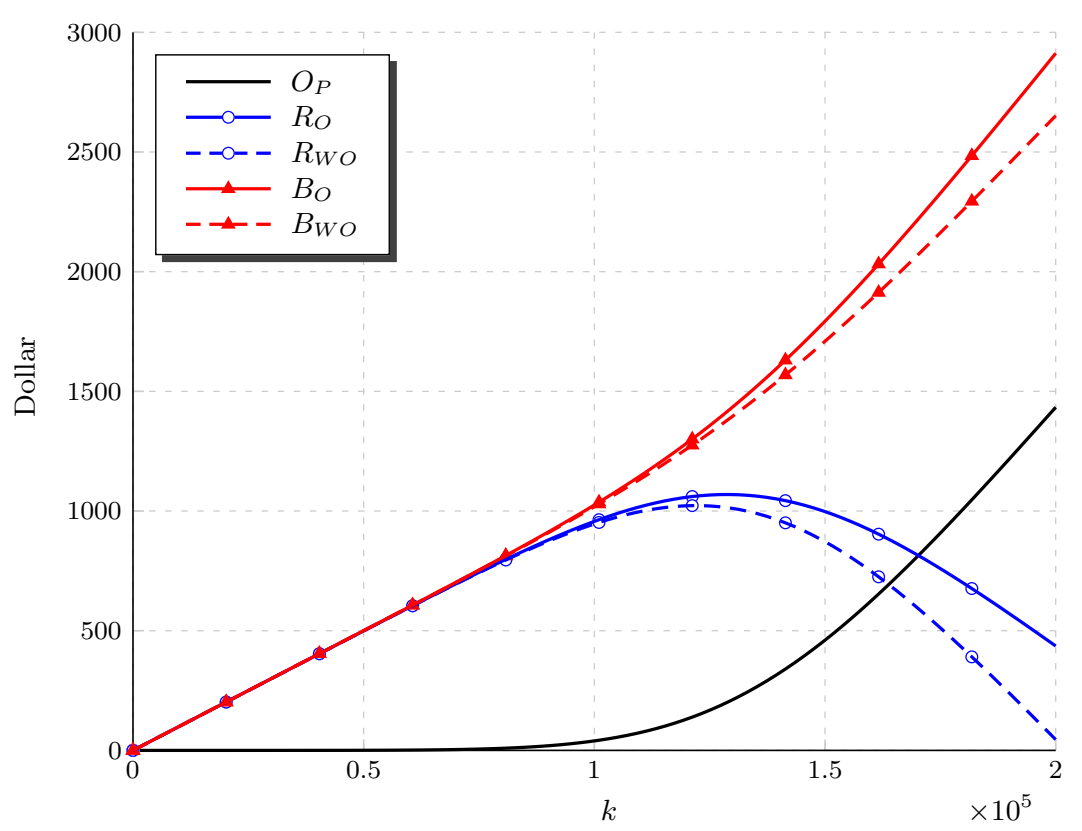

Figure 1: Option Price, Revenues, and Benefits versus Contracted number of displays

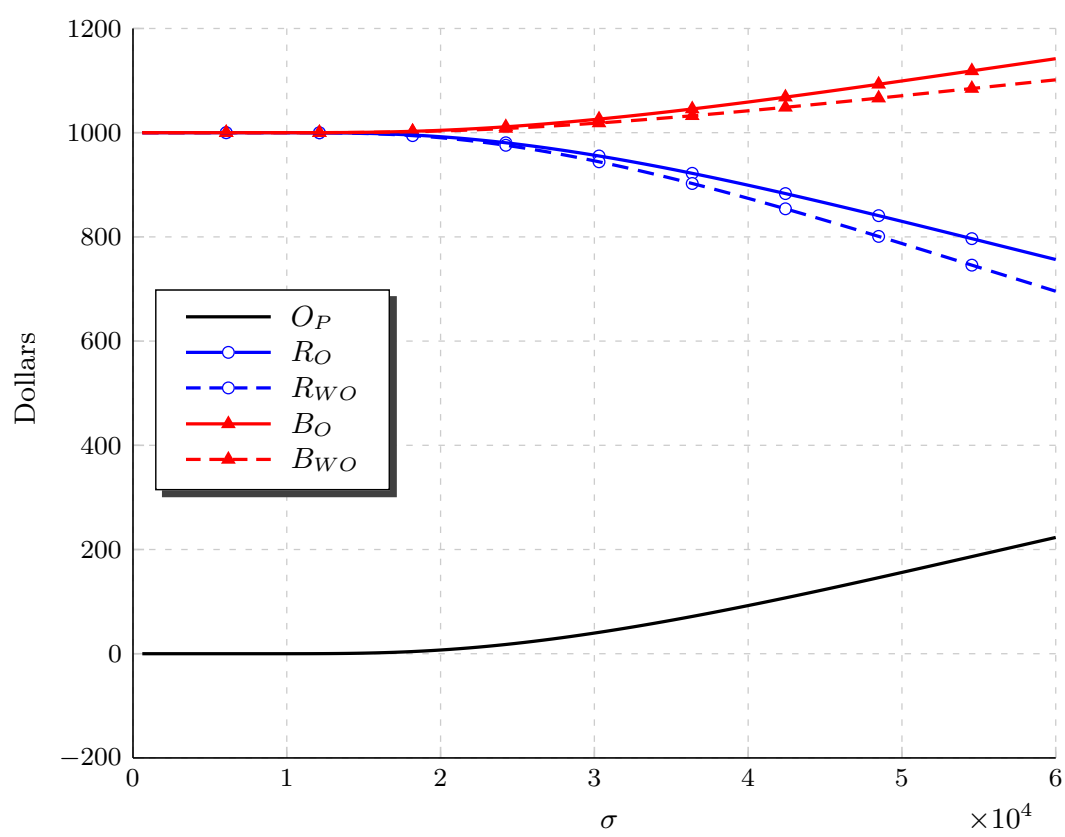

Figure 2: Option Price, Revenuews, and Benefits versus Standard Deviation 


\subsection{Effects of the Standard Deviation of the Number of Page- Views}

The effects of the standard deviation of the number of page-views in Page 1 are considered. If the standard deviation increases, it becomes riskier to promise a certain number of displays; therefore, the option contract, which can be thought as an insurance that protects the publisher, has more value to the publisher. Therefore intuitively, the option price should increase as the variance increases. Because $\frac{\partial \gamma}{\partial \sigma}$ is rather difficult to quantify for general cases, we first consider a simple uniform distribution case on the interval $[a, b]$. Then $\mathbb{E}\left[X_{1}\right]=\mu=(a+b) / 2$ and the standard deviation of $X_{1}$ is $\sigma=(b-a) / \sqrt{12}=\delta / \sqrt{3}$ where $a=\mu-\delta$ and $b=\mu+\delta$. For $k \in[a, b]=[\mu-\delta, \mu+\delta]$, we have

$$
\gamma=k F_{1}(k)-\int_{0}^{k} x f_{1}(x) d x=\frac{(k-\mu+\delta)^{2}}{4 \delta}
$$

and

$$
\frac{\partial \gamma}{\partial \delta}=\frac{(\delta-k+\mu)(k-\mu+\delta)}{4 \delta^{2}} \geq 0
$$

Therefore

$$
\begin{aligned}
\frac{\partial O_{P}}{\partial \sigma} & =\frac{\partial O_{P}}{\partial \gamma} \frac{\partial \gamma}{\partial \delta} \frac{\partial \delta}{\partial \sigma} \\
& =\left(p_{2}+h-\alpha U_{2}^{\prime}(\gamma)\right) \frac{(\delta-k+\mu)(k-\mu+\delta)}{4 \delta^{2}} \sqrt{3}
\end{aligned}
$$

Since $k \in[a, b]=[\mu-\delta, \mu+\delta]$ and $p_{2}+h-\alpha U_{2}^{\prime}(\gamma) \geq 0$ from (11), we have $\frac{\partial O_{P}}{\partial \sigma} \geq 0$.

In Figure 2, the changes in the option price, revenues and benefits as the standard deviation changes are presented, where we used Normal distributions; all other parameters remain same. We observe that the option price increases with increasing $\sigma$ as we can anticipate. The expected revenues of the publisher decrease because of higher chance of paying high penalty (without option) or receiving less revenue (with option). The expected benefits of the advertiser, on the other hand, increase because of the same reason; the advertiser needs to pay less.

\section{Realised Page-Views and Benefit of the Option Contract}

The analysis so far has been on the expected values. In this section, we study the cases when revenue without the option actually turns out to be higher than the revenue with the option. We define the revenues for the publisher and the benefits of advertising to the advertiser in terms of the realised page-view as opposed to the probability 
distribution as done earlier. We define $x$ to be the actual number of page views on Page 1, realised on any given day. All other variables defined as before. Then the revenue with the option becomes

$$
R_{O}=p_{1} \min (x, k)+p_{2} \max (k-x, 0)-O_{P}
$$

Similarly, we have

$$
\begin{aligned}
R_{W O} & =p_{1} \min (x, k)-h \max (k-x, 0) \\
B_{O} & =U_{1}(\min (x, k))+U_{2}(\max (k-x, 0))-R_{O} \\
B_{W O} & =U_{1}(\min (x, k))-R_{W O}
\end{aligned}
$$

Using this form of defining the revenues and benefits allows us to compare the hypothetical revenues and benefits arising from a realised value of the number of page views on Page 1. The exact number of page views which results in revenue with the option to be greater than revenue without options can be obtained analytically as follows. From the condition $R_{O}>R_{W O}$, we obtain

$$
p_{1} \min (x, k)+p_{2} \max (k-x, 0)-O_{P}>p_{1} \min (x, k)-h \max (k-x, 0)
$$

and, consequently,

$$
x<k-\frac{O_{P}}{p_{2}+h} \equiv \hat{x}
$$

This indicates that any value of $x<\hat{x}$ will result in $R_{W O}<R_{O}$ for the publisher. That is, if the number of page-views in Page 1 turns out to be too small, the publisher would benefit from an option contract.

A similar analysis for benefits to the advertiser with and without options is carried out as follows. From the condition $B_{O}>B_{W O}$, we obtain

$$
U_{1}(\min (x, k))+U_{2}(\max (k-x, 0))-R_{O}>U_{1}(\min (x, k))-R_{W O}
$$

and, therefore

$$
U_{2}(k-x)+\left(p_{2}+h\right) x>\left(p_{2}+h\right) x-O_{P}
$$

If we employ the linear utility functions of the advertiser as done in the previous section, we obtain

$$
x>k-\frac{O_{P}}{p_{2}+h-m_{2}} \equiv \bar{x}
$$

That is, if the number of page-views in Page 1 is realised greater than $\bar{x}$, the advertiser would benefit from an option contract. We can understand this result as in the 


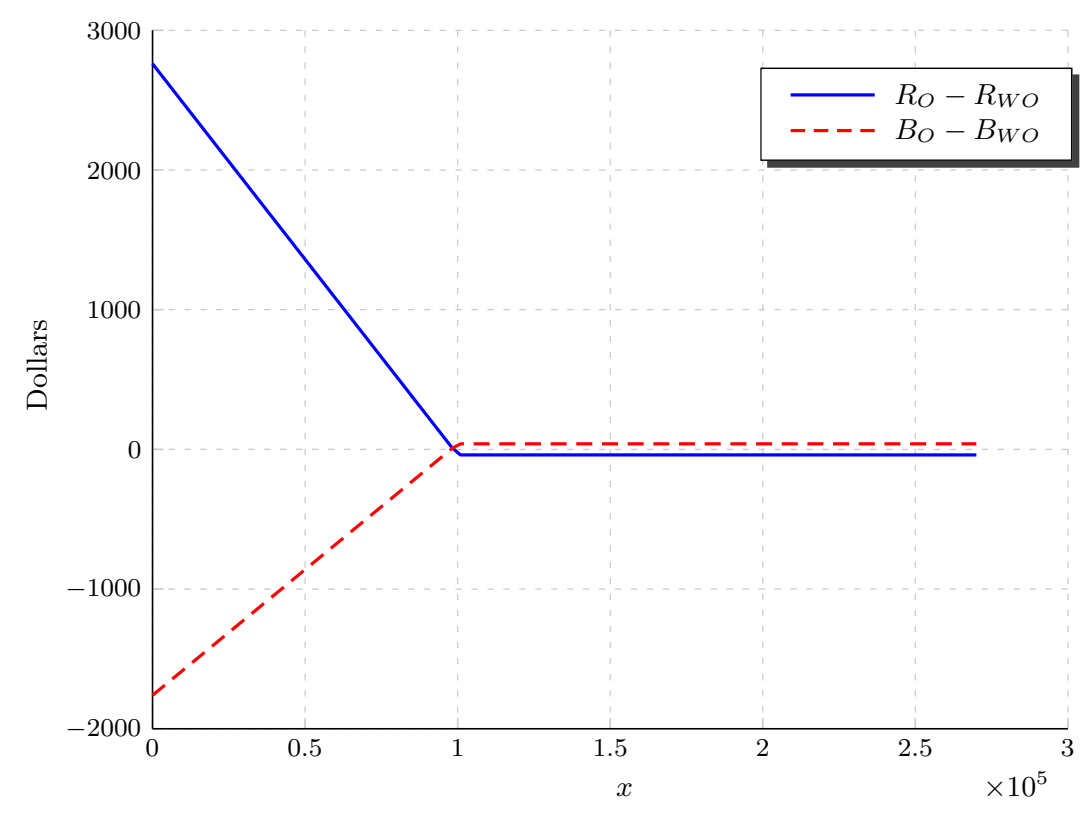

Figure 3: Revenues and benefits with realised numbers of page-views on Page 1

following way. The advertiser's primary objective is to advertise in Page $1 k$-times. Therefore if the page-view in Page 1 is sufficiently large, the target objective is reached and the advertiser collected the option price from the publisher, or received discounts as much as the option price.

We further test these values using numerical studies. We first compute the option price $O_{P}=39.63$, using a data from Table 1 , and compute the values of revenues and benefits. We present the differences $R_{O}-R_{W O}$ and $B_{O}-B_{W O}$ with realised $x$ values in Figure 3. When $x$ is sufficiently large, the publisher loses exactly $O_{P}=39.63$ while the advertiser gains $O_{P}=39.63$.

In Figure 3, it looks like the advertiser has no incentive to enter into an option contract because the advertiser benefits much more with an option contract. However, the probability that the advertiser is more beneficial is much greater than the publisher. To illustrate this, using the values from Table 1, we compute the probabilities when the publisher and the advertiser are more beneficial.

We first obtain $\hat{x}=98,846$ and $\bar{x}=97,798$. Note that the contracted number of displays is $k=100,000$. The values of $\hat{x}$ and $\bar{x}$ effectively become the upper and lower limits for the region of $x$ where both the publisher and the advertiser benefit by using the option. The probability that the number of page views on Page 1 falls between these values of $\hat{x}$ and $\bar{x}$ can be an important factor in the decision choice for the advertiser and the publisher. The values of $\hat{x}$ and $\bar{x}$ are based on data available to the publisher and advertiser before entering into the contract for advertising. Thus it is possible for the publisher and the advertiser to compute these values and carry out the 


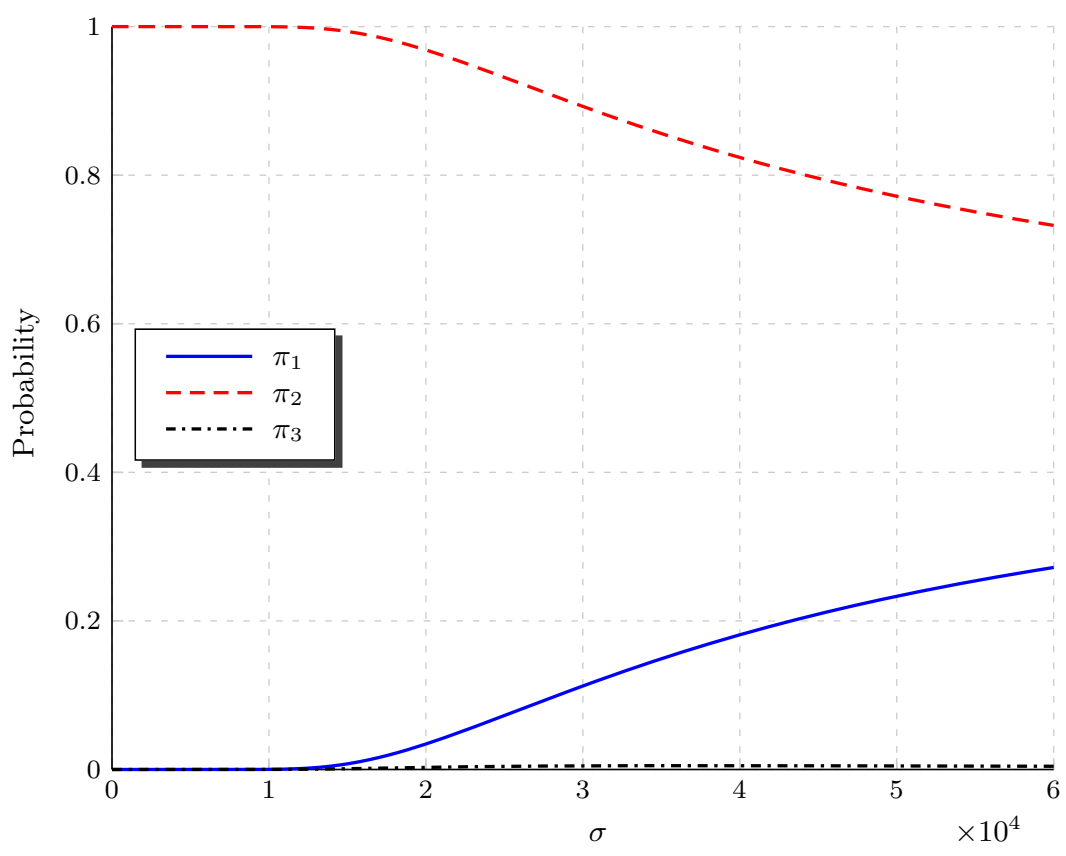

Figure 4: Impact of standard deviation on the probability of profitability of the option for the publisher and the advertiser

following analysis before entering into the contract. The upper limit $\hat{x}$ corresponds to the publisher's region of profitability while the lower limit $\bar{x}$ corresponds to the advertiser's region of profitability.

We study the impact of variation in standard deviation and the contracted number of displays on the probabilities defined above. The results are provided in Figures 4 and 5, respectively. We define the following three probabilities:

$$
\begin{aligned}
& \pi_{1}=\operatorname{Pr}(X<\hat{x})=F_{1}(\hat{x}) \\
& \pi_{2}=\operatorname{Pr}(X>\bar{x})=1-F_{1}(\bar{x}) \\
& \pi_{3}=\operatorname{Pr}(\bar{x}<X<\hat{x})=F_{1}(\hat{x})-F_{1}(\bar{x})
\end{aligned}
$$

where $\pi_{1}$ is the probability that the publisher benefits from the option, $\pi_{2}$ is the probability that the advertiser benefits, and $\pi_{3}$ is the probability that both benefit. We can observe that the advertiser benefits more often than the publisher. That is, the advertiser can save the advertising fee (by the amount of the option price) with the option contract, while the publisher can protect the revenue system from a small number of page-views. This result indicates that the option contract is indeed a protection insurance for the publisher. 


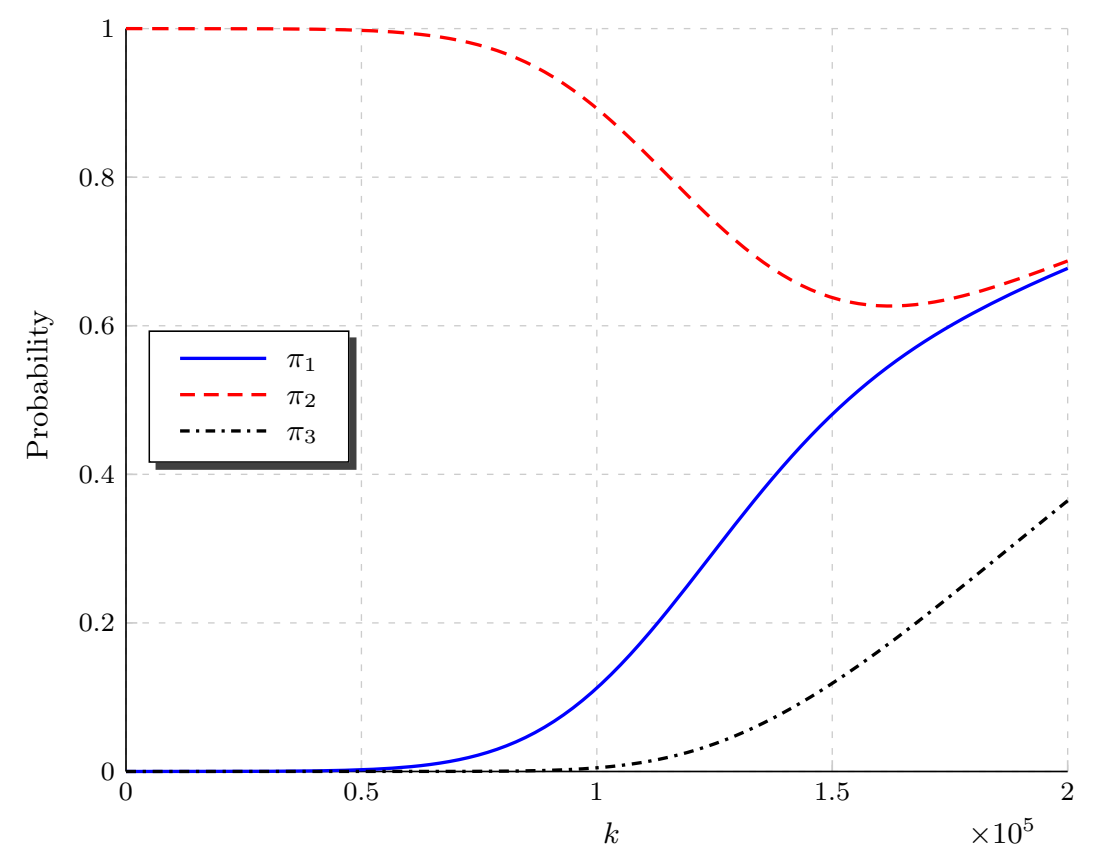

Figure 5: Impact of initially contracted number of displays on the probability of profitability of the option for the publisher and the advertiser

\section{Conclusion}

For any business, uncertainty always has an impact on the revenues. The use of options in such scenarios helps controlling this risk to some extent. In online advertising, this risk is particularly high as the number of page-views depends on a variety of factors as discussed in several research papers. In this article, we look at a specific case in the online advertising and provide a way to mitigate the risk of uncertain number of page-views. We have assumed here that the advertiser is more interested in receiving the advertising benefits than the penalty cost. The surplus created from the option, increases with increase in standard deviation in the probability distribution for the number of page-views. The higher the standard deviation, the more volatile is the demand and thus the option should, by intuition provide higher benefits. The results follow this intuition. The option price decreases with increasing relative negotiation powers of the publisher. Also, the contracted number of displays of advertisement on Page 1 has an impact on the option price. The higher this number,the greater is the risk from a fluctuating number of page views and hence greater is the option price. Another implication of the results is that the option creates a win-win situation for the publisher and the advertiser only if the advertiser gets some advertising effect from advertising on Page 2. Otherwise, the surplus created by the option for the publisher would be nil.

We have shown that the proposed option contract can be beneficial to both the 
publisher and the advertiser. Since the publisher is exposed to uncertain number of page-views, he can face a large amount of revenue loses when the number of page-views is realised at low level. The publisher will likely lose the option price, but he obtains a protection as in health or auto insurances. On the other hand, the advertiser can reduce the advertisement fee with a high probability with the option contract.

The option contract presented in this paper is designed in such a way that the option can be exercised only at the end of the period for which the initial contract has been made. If exercised by the publisher, the unfulfilled displays will be published on Page 2 in the subsequent period. A limitation of this model is that the publisher needs to have two web-pages between which he can shift advertisements and the viewership of the two pages needs to be similar in terms of number of viewers and viewer demography. A web-page need not necessarily mean one page of the website. The same model can be applied if the web-pages are replaced by two pages on the same site or by two networks. Also, the model is based on the assumption that the advertiser is more interested in receiving the advertising effect than the penalty from the publisher. This may not necessarily be the case with every advertiser. The proposed model introduces option contracts between a publisher who has two web-pages and the advertiser.

In some cases, a publisher maintains multiple numbers of web-pages, but the option contract proposed in this paper can model only two pages. Therefore, an extension of the same model could be developed for a publisher who has more than three web-pages, so that shifts between pages can be multi-level and specific to advertisers' preferences. Also the current model introduces option contract which can be exercised only once at the end of the period. One could also consider using American style options which could be exercised anytime within a specific time period. Such American style options require dynamic modeling of page-views.

\section{References}

Becker, H., C. Meek, and D. M. Chickering (2007). Modeling contextual factors of click rates. In Proceedings of the 22nd National Conference on Artificial Intelligence, Volume 2, pp. 1310-1315. AAAI Press.

Chatterjee, P., D. L. Hoffman, and T. P. Novak (2003,). Modeling the Clickstream: Implications for Web-Based Advertising Efforts. Marketing Science 22(4), 520-541.

Ching, W.-K., X. Li, T. K. Siu, and Z. Wu (2010). Improving Revenue Management: A Real Option Approach. In P. Bernus, J. Błażewics, G. Schmidt, M. Shaw, T. C. E. Cheng, and T.-M. Choi (Eds.), Innovative Quick Response Programs in Logistics 
and Supply Chain Management, International Handbooks on Information Systems, Chapter 6, pp. 123-139. Berlin, Heidelberg: Springer Berlin Heidelberg.

Danaher, P. J. and G. W. Mullarkey (2003). Factors Affecting Online Advertising Recall: A Study of Students. Journal of Advertising Research 43(3), 252-267.

Dinev, T., Q. Hu, and A. Yayla (2008). Is There an On-line Advertisers' Dilemma? A Study of Click Fraud in the Pay-Per-Click Model. International Journal of Electronic Commerce 13(2), 29-60.

Drèze, X. and F.-X. Hussherr (2003). Internet advertising: Is anybody watching? $J$. Interactive Mark. 17(4), 8-23.

Elmaghraby, W., S. A. Lippman, C. S. Tang, and R. Yin (2009). Will More Purchasing Options Benefit Customers? Production and Operations Management 18(4), 381401.

Evans, D. S. (2008). The Economics of the Online Advertising Industry. Review of Network Economics 7(3), 359-391.

Fjell, K. (2009). Online advertising: Pay-per-view versus pay-per-click - A comment. Journal of Revenue \& Pricing Management 8, 200-206.

Gallego, G., S. G. Kou, and R. Phillips (2008). Revenue Management of Callable Products. Management Science 54(3), 550-564.

Gallego, G. and R. Phillips (2004). Revenue Management of Flexible Products. Manufacturing \& Service Operations Management 6(4), 321-337.

Internet Advertising Bureau (2010). IAB Internet Advertising Revenue Report. Technical report, Internet Advertising Bureau.

Kumar, S. and S. P. Sethi (2009). Dynamic pricing and advertising for web content providers. European Journal of Operational Research 197(3), 924-944.

Kwon, C. (2009). Single-Period Balancing of Pay-Per-Click and Pay-Per-View Online Display Advertisements. Journal of Revenue and Pricing Management. In press.

Mahdian, M. and K. Tomak (2008). Pay-Per-Action Model for On-line Advertising. International Journal of Electronic Commerce 13(2), 113-128.

Mangani, A. (2004). Online advertising: Pay-per-view versus pay-per-click. Journal of Revenue and Pricing Management, 295-302. 
McCoy, S., A. Everard, P. Polak, and D. F. Galletta (2007). The effects of online advertising. Communications of the ACM 50(3), 84-88.

Moon, Y. and C. Kwon (2011). Online advertisement service pricing and an option contract. Electronic Commerce Research and Applications 10(1), 38-48.

Nagarajan, M. and G. Sosic (2008). Game-theoretic analysis of cooperation among supply chain agents: Review and extensions. European Journal of Operational Research 187(3), 719-745.

Nash Jr., J. (1950). The Bargaining Problem. Econometrica 18(2), 155-162.

Richardson, M., E. Dominowska, and R. Ragno (2007). Predicting clicks: estimating the click-through rate for new ads. In Proceedings of the 16th international conference on World Wide Web, WWW '07, New York, NY, USA, pp. 521-530. ACM.

Roels, G. and K. Fridgeirsdottir (2009). Dynamic revenue management for online display advertising. Journal of Revenue and Pricing Management 8(5), 452-466.

Roth, A. E. and M. W. Malouf (1979). Game-theoretic models and the role of information in bargaining. Psychological Review 86(6), 574-594.

Touati, C., E. Altman, and J. Galtier (2006,). Generalized Nash Bargaining Solution for Bandwidth Allocation. Computer Networks 50(17), 3242-3263. 